\title{
Patient Satisfaction on Post Operative Instructions Given by Hospital Staff on Discharge
}

Tennekoon D., McPartlin D., Beynon Philips A., Tadikonda R., Arotiowa M.

\section{Introduction.}

Patient satisfaction plays an important role in the health care process. It influences the health care seeking behaviour of patients, compliance with treatment and the outcome of the patient. This is important as it ensures the good quality of care, communication and finally better clinical outcomes- which are key factors in a successful health care system. Since early 1980 s the interest in the studies on patient satisfaction with their health care experience has increased. This followed reports that patient satisfaction is associated with better clinical outcomes. This has not been universally recognized. However, patient satisfaction is regarded as a quality of life care marker all over the word. ${ }^{1}$.

Level of satisfaction will be unique to each individual, and also depends on the type of information they have been provided with. A key but mostly disregarded aspect of post-operative care occurs after the patient is discharged following surgery.

When considering the factors that affect patient satisfaction, it has been noted that patients show

concern during the post-operative period about ${ }^{2}$

a) Post-operative complications and their management

b) Concerns about conditions deteriorating after discharge

c) Instructions given (written and verbally) in relation to the anticipated recovery at home

The patient who is given proper and adequate instructions will develop a correct and positive attitude during recovery. They will willingly be following instructions making the recovery period problem free, which will lead to a rapid recovery. Giving them appropriate knowledge can make them change their attitudes, beliefs and behaviour towards the illness, treatment and the period of recovery. ${ }^{2}$

A significant number of patient satisfaction studies exist but only a few are considered as valid and reliable. Also a very few studies have addressed patient satisfaction on post-operative instructions given by doctors on discharge. A literature survey done has failed to demonstrate any published studies on patient satisfaction on post-operative instructions given following Ear, Nose and Throat surgeries. ${ }^{8}$

\section{Material and Methods.}

\section{Study objective}

The main objective of the study is to evaluate patient satisfaction on post-operative instruction given by medical staff on discharge following ear, nose and throat surgeries and secondarily, to develop guidelines for verbal and written instructions pertaining to the anticipated recovery at home following Ear, Nose and Throat surgeries.

\section{Design}

The study was a prospective observational study with questionnaires given on discharge of the patient following ENT surgeries. 3 separate questionnaires on Ear, Nose, Throat and Neck surgeries were distributed among the patients. The questionnaires were based on a previously validated instrument slightly modified to reflect satisfaction with regards to ENT surgeries.

Each type of questionnaire had 5 variable questions: 


\section{Nasal}

Patient satisfaction regarding instructions given on

A) General post-operative complications like pain, nausea, vomiting.

B) Post-operative activities like nasal douching, nose blowing, sneezing, stent/ suture removal.

C) Complications of nasal surgery like pain, nasal obstruction, nasal bleeding, crust formation, loss of smell and, in major surgeries, eye complications.

D) Medication given on discharge (Name of the drug, purpose, dose and frequency)

E) Further plan of management

\section{Ear}

Patient satisfaction regarding instructions given on

A) General post-operative complications like pain, nausea, vomiting and dizziness.

B) Post-operative activities like dressing removal, pack removal, suture removal, straining, hair washing and driving.

C) Post-operative common symptoms like ear fullness, pain, ear discharge, vertigo, tinnitus and change in hearing.

D) Medication given on discharge (Name of the drug, purpose, dose and frequency)

E) Further plan of management

\section{Throat and Neck}

Patient satisfaction regarding instructions given on

A) General post-operative complications like pain, nausea, vomiting, hoarseness of voice, difficulty in swallowing and bleeding.
B) Post-operative activities like dressing change, voice rest, swallowing, speech therapy and drain/suture removal in neck surgeries.

C) Complication of the surgery like loss of voice, change in voice, neck scar, swelling at surgery site, bleeding, numbness of face/ ear in parotid surgeries and nerve damage in salivary gland surgeries.

D) Medication given on discharge (Name of the drug, purpose, dose and frequency)

E) Further plan of management

There were 5 general questions given at the end of each questionnaire:

1) Patient's comments or suggestions on any of the topics

2) Overall rating on services provided by the ENT unit

3) Whether a patient would recommend the hospital to friends and family

4) Main reason for giving the above scores

5) What has to be done to enable a better score

The questionnaires were distributed on postoperative day one or day 2 before discharge. Some patients required the assistance of the hospital staff when filling out the questionnaire and the responses of the participants under the age of 12 were given by their parents. Once completed the questionnaires were collected and filed separately; dividing them into minor, intermediate and major surgeries in the respective categories.

Each rating question was accompanied by a visual analog scale with number ranging from 1 to 9 , with 1 being poor and 9 being good, for the patient to easily express their satisfaction. When analysing the results, the numbers from 1-3 were taken as 'Poor', 4-6 as 'Satisfactory' and 7-9 as 'Good'. 
Data collection and analysis were done by the author with the help of the research/audit department of QEH, Kings Lynn.

\section{Setting}

The Queen Elizabeth Hospital, Kings Lynn, from 1st January 2016 - 1st January 2017

\section{Subjects}

Patients undergoing minor, intermediate and major ENT procedures under anaesthesia were involved in the study. Subjects were informed about the study and its aims, confirming that identification would remain anonymous and that the results were confidential.

\section{Results \& Discussion}

The survey collected information from 160 participants, which included 81 males and 79 females, ages ranging from 2 Years to 80 years.

\section{Nasal surgeries}

52 patients (30 females and 22 males) underwent Nasal surgeries including minor nasal surgeries (nasal bone fracture reduction; excision of nasal lesions and septal button insertions), intermediate nasal surgeries (septoplasty; endoscopic nasal polypectomy and middle meatal antrostomys) and major surgeries (septorhinoplasty; advanced FESS and DCR surgeries). Out of 10 patients who underwent minor nasal surgeries 5 were male and 5 were females. There responses for the 5 variable questions are as follows (Fig. 1)

As none of the responses included 'Poor' as a rating, it has been omitted in the chart. There was an overall positive response towards the topics raised in questions $\mathrm{A}$ (General post-operative complications), B (Post-operative activities), $\mathrm{C}$ (Complications) and D (Medication), with a majority being 'Good'), however, the response towards the question $\mathrm{E}$ (regarding further management plans) were not as positive (as a majority rated it as 'Satisfactory'). The data shows that male patients were generally more likely to give a rating of 'Good', whereas females were more likely to rate the services as 'Satisfactory' especially on medication given on discharge.

The responses for the 5 variable questions of the 12 patients who underwent Intermediate nasal surgeries ( 7 males and 5 females) are as follows (Fig. 2) Again 'Poor' has been omitted as none of the participants gave a rating of 'Poor'. A majority of the responses were positive on questions $\mathrm{B}$ (post-operative activities), C (complications of surgery) and $\mathrm{E}$ (further management plan). Again, male participants showed a more positive response than females in all the 5 variable questions.

When analysing data on post-operative instructions given following Major nasal surgeries, the responses of the 30 patients (14 males and 16 females) are as follows (Fig. 3) Unlike the results for the Minor and Intermediate nasal surgeries, one patients out of 30 responded with a rating of 'Poor' for Question A (Instructions on General post-operative instructions). There was an overall positive response for all the 5 variable questions, again with male participants showing a higher rating that female participants.

The overall rating for information given on discharge following Minor, Intermediate and Major Nasal surgery is depicted in the pie chart below. (Fig. 4) $77 \%$ of the responses regarding the Post-operative nasal surgeries showed a rating of 'Good', 21\% a rating of 'Satisfactory' and 2\% a rating of 'Poor', with the rating for 'poor' being a single patient regarding instructions given on general post-operative complications following major nasal surgery.

\section{Ear Surgery}

53 participants underwent Ear surgeries including Minor surgeries like Myringotomy and Grometts; Intermediate surgeries like Tympanoplasty, Pinaplasty and Canaloplasty; and Major surgeries like Combined Approach Tympanoplasty (1st and 2nd look), Ossiculoplasty, Tympano and Canaloplasty, Stapes surgery and Mastoid 
obliteration surgery. 21 participants underwent Minor ear surgeries (14 males and 7 females). Their responses to the 5 variable questions were as follows (Fig. 5)

For all 5 variable questions the majority of the ratings were 'Good', with a low but consistent number responding as 'Satisfactory' to each question. Again, 'Poor' has been omitted as there were no ratings as 'Poor'. Proportionally all males and females have given similar ratings. 8 participants underwent Intermediate ear surgeries (5 males and 3 females). Their responses were as follows (Fig. 6)

Questions A, C, D and E received a unanimous rating of 'Good' by both male and female respondents, white question B (advice on postoperative activities) received a single rating of 'Satisfactory'. Again, 'Poor' has been omitted as there were no ratings as 'Poor'. Proportionally all males and females have given similar ratings. The responses of the 24 participants who underwent Major ear surgeries (12 male and 12 female) were as follows (Fig. 7)

With the exception of a single female respondent giving the rating as 'Satisfactory' on question A (General post-operative complications), all the other participants gave a unanimous rating of 'Good' for all the questions. When considering ear surgeries (minor, intermediate and major) the overall response from the patients was 'Good', especially in the major ear surgery category as the patient satisfaction showed the highest score. The overall response for ear surgeries can be depicted as follows, with $92 \%$ responding as 'Good' and the remaining 8\% responding as 'Satisfactory'. (Fig. 8)

\section{Oropharynx and Neck surgery}

The Minor surgeries involved were simple neck lump excision, tongue biopsies and biopsy of skin lesions in the neck. When analyzing data on Minor Oropharyngal and Neck surgeries of 10 patients' ( 8 males and 2 females) the following results were obtained. (Fig. 9)
All the patients responded as 'Good' for questions A (General post-operative complications) and C (Explanations of complications following surgery). However, questions $\mathrm{D}$ and $\mathrm{E}$, which gave explanations

about medications after surgery and the future plan of management, didn't show as much of positive response when compared to $\mathrm{A}$ and $\mathrm{C}$, with half responding as 'Good' and the other half as 'Satisfactory'. None of the patients responded with 'Poor'. Males and females have shown a similar type of responses. The surgeries involved in Intermediate Oropharynx and Neck surgeries were: Tonsillectomies, Microlaryngoscopy/ Pharyngoscopy and biopsy and Adenoidectomy. The responses of the 37 patients (15 males and 22 females) included in this group are as follows (Fig, 10)

A majority of the patients in this category responded with 'Good' for the questions A, B and C (regarding general post-operative complications, explanation of post-operative activities and complications after surgery). However, questions $\mathrm{D}$ and $\mathrm{E}$ (regarding medication on discharge and future management plan) did not show as positive results as the others. In contrast to other surgeries involving ear, nose and orapharyngal minor, the intermediate oropharyngeal category showed the response 'Poor' to questions $\mathrm{D}$ and $\mathrm{E}$, and both responses were made by females.

The surgeries involved in Major Oropharynx and neck surgeries were: excision of salivary gland tumor, branchial (only one patient) and thyroglossal cyst excision (one patient). The responses of the 8 patients ( 1 male and 7 females) were as follows. (Fig. 11)

Questions A, B and C (regarding general postoperative complications, advise on post-operative activities and complications of surgery) showed a unanimous result as 'Good' by both males and females. However, the results show stark differences in the responses to the questions $\mathrm{D}$ and $\mathrm{E}$ (regarding medication given on discharge 
and future management plan) with only the male patient responding as 'Good' and all the female participants responding as 'Satisfactory'. When analysing the overall rating on minor, intermediate and major oropharyngeal and neck surgeries; 73\% responded with 'Good', 25\% responded with 'Satisfactory' and 2\% responded with 'Poor'. (Fig. 12)

\section{General Comments Assessment}

In the questionnaire a section was allocated for the patient's comments for improvements and suggestions about the post-operative instructions. The responses for these following ear, nose and oropharynx and neck surgeries were analysed all together. The findings are summarized as follows.

1) The majority of the patients said there should be more time allocated for doctorpatientcommunication following the postoperative period before discharge.

2) More time should be allocated for the patient to ask questions.

3) Presence of family members during this post-operative meeting.

It was also noticed that some patients were aware of the pressure on doctors and were reluctant to ask questions. Although a few patients did not respond to the question on why they gave their relevant scores, the ones that did, said that it was because the staff was friendly and that they were generally happy about the services provided.

On the question on whether the patient would recommend the hospital 99\% responded saying "highly likely", giving a score of 9 on the visual analog scale, while the remaining $1 \%$ responded saying "likely, giving a score of either 7 or 8 . On the final question on what the hospital needed to do to enable the patient to award them the highest score, their responses can be summarized as.

1) Patients reported that they did not know if one doctor was in overall charge of their care orwhether there were several doctors.
Some recommended producing a sheet of information orphoto identity of doctors and medical staff with easy to read name badges.

2) Patients and relatives should have opportunities to ask questions and to explain what is on theirminds.

3) Patients should be given information at the right time when they can absorb it, rather thanimmediately after surgery when the effects of anaesthesia are still present.

4) Information that is easy to understand/ without much medical jargon.

5) All the medical staff should provide the same information as it is disturbing to receiveconflicting advice.

\section{Conclusion}

The purpose of this study was to find out the patient satisfaction on post-operative instructions given by medical staff/doctors on discharge. This gave us an idea whether the specific patient had an aftercare plan in place before leaving the hospital. Since satisfied patients tend to comply with medical treatment; less likely to go doctor shopping; and ultimately a successful outcome. Even though the satisfaction is a subjective judgement, this study has given important information for the health care providers to produce better outcomes.

The study reveals that a majority of the patients that underwent ear, nose and oropharynx and neck surgeries were happy about the postoperative instructions they received following their respective surgeries, especially regarding the instructions they received on general postoperative complications following surgery (Question A) and the complications of surgery (Question C). On instruction on postoperative activities (Question B) patients were generally satisfied but there is room for improvement.

There was a contrast in patient satisfaction on the instructions regarding medication given 
Tennekoon D., McPartlin D., Beynon Philips A., Tadikonda R., Arotiowa M. Patient Satisfaction on Post Operative Instructions Given by Hospital Staff on Discharge Volume : 6; Issue1; pp 15-22

on discharge (Question D) and the further management plan (Question E) following Oropharyngeal and neck surgeries (minor, intermediate and major) as a significant amount of patients responded as 'Satisfactory' and 'Poor'.

Patient satisfaction seemed to be highest on postoperative instructions following ear surgeries, out of which the results for Major ear surgeries showed the highest satisfaction rate of all the categories. Input from patients for the open ended general question gives a good insight into the improvements that can be made to ensure a high level of patient satisfaction, thereby, better patient compliance; leading to achieving better results.

\section{Limitations}

This study improves our understanding of patient satisfaction and provides useful information regarding post-operative instructions. However, it has some limitations. As the doctors participating in the study were aware of their role in the study, towards the end of the study they were well versed in their role and were conversing better with the patients. The fact they were aware of their role could have influenced their practice during this study period.

The doctors had to rely on their knowledge when conveying the information to the patients before discharge. As there are no current guidelines on post-operative ENT instructions and since all the doctors were not at an equal level in qualification, the instructions given were not standardized. Therefore, it is likely that all aspects and procedures were not covered by the doctors resulting in different scores from patients. The questionnaires of the paediatric patients were filled by the parents and the questionnaires of elderly by the hospital staff. This could have had a minor impact on the scores received.

\section{References.}

1. Emmanuel K.Patient Satisfaction studies and monitoring of Right to Health: Global
Journal of Health andScience Vol. 3, No. 1, Faculty of Medicine, GenevaUniversity; 2011

2. Koichiro O.Patient Satisfaction focusing on "Excellent", Ref. \#07010005884,Purdue University

3. Factors affecting parental satisfaction following paediatric procedural sedation: PMC J Cin Anaesth22(1)29134; 2010

4. A qualitative report of patient problems and post-operative instructions - Oro maxillofacialSurgery,PMC. J. oral fac surg, 63(4)449-456; 2014

5. Oliveria D. The impacts of health literacy in the case of surgical patients. A qualitative systematic review, BMC Surgery,BMC 15.86Doi 10.118615 $1289315 \quad 0073-6$; 2015

6. How creation of Parent Satisfaction Questionnaire Improved Multidisciplinary Service Deliveryin a Pedriatric Day Surgery Unit.BMJ QUAL improve report. 2 cloi 10.1136; 2013

7. Post-Operative Instructions ENT Surgeons of Western NewEngland. SPRINGFIELD 413-732-7426

8. Good Medical Practice. General Medical Council (GMC); 2017

9. Good Surgical Practice. RCS (England); 2008

10. Patient Satisfaction - Why Should We Care? [Internet]. Medscape. 2017. Available from: http://www.medscape. com/viewarticle/736495

11. Ndambuki J. The level of patients' satisfaction and perception on quality of nursing service in the Renal unit,Kenyatta National Hospital Nairobi, Kenya: Open Journal of Nursing, Vol 3, 186-194; 2013 


\section{Tables}

\section{Figures and Illustrations.}

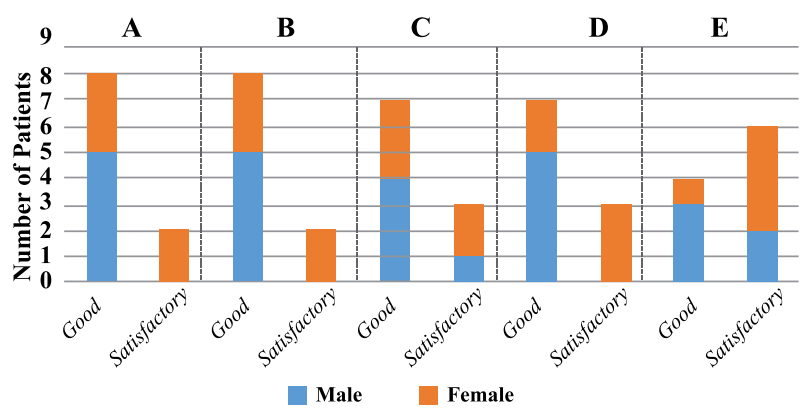

Fig 1- Results of Minor Nasal Surgeries

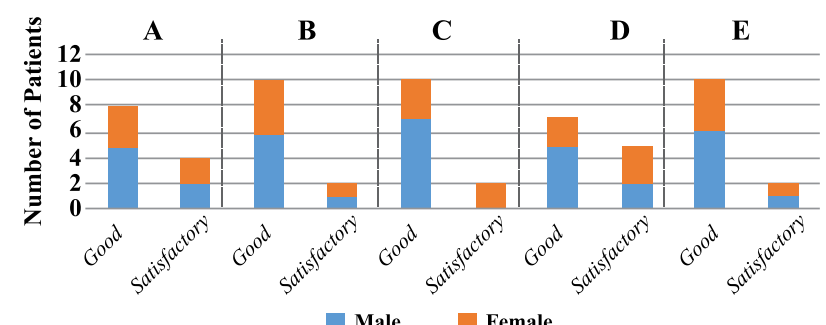

Fig 2- Results of Intermediate Nasal Surgeries

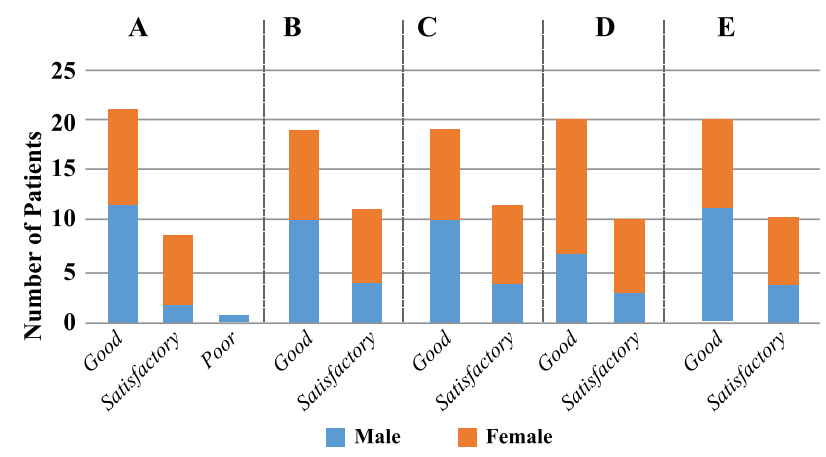

Fig 3- Results of Major Nasal Surgeries

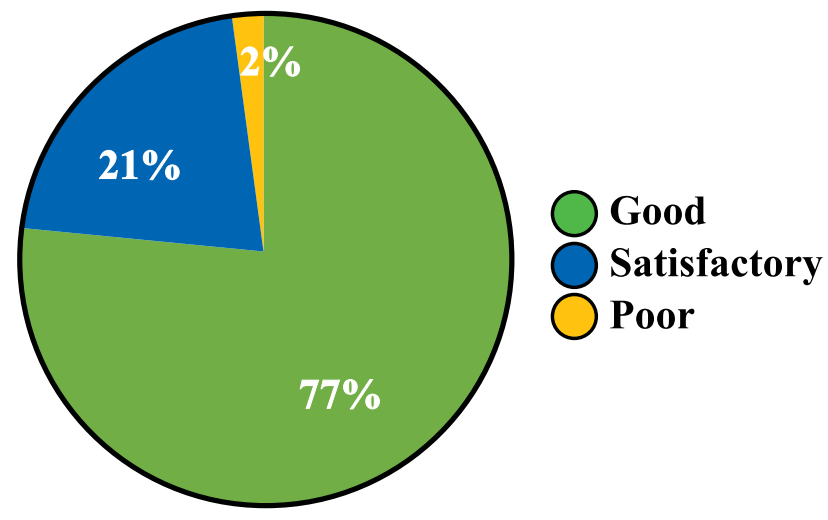

Fig 4 - Overrall score of Nasal Surgeries

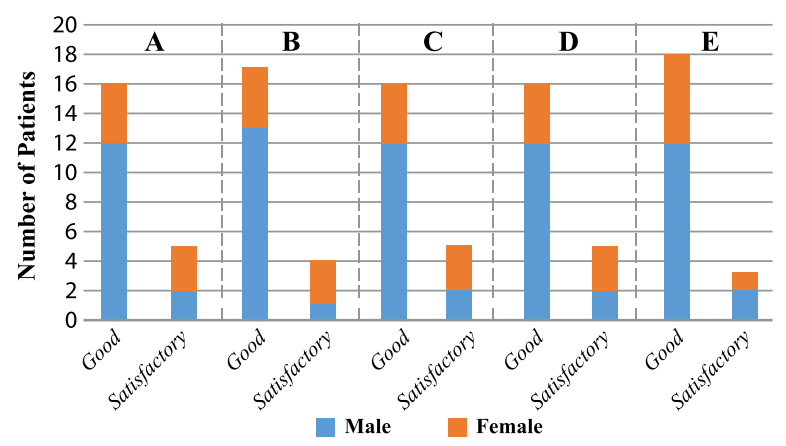

Fig 5 - Results of Minor Ear Surgeries

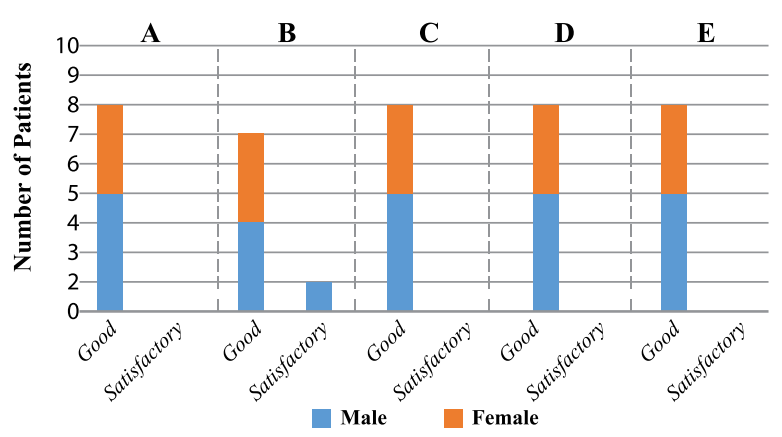

Fig 6 - Results of Intermediate Ear Surgeries

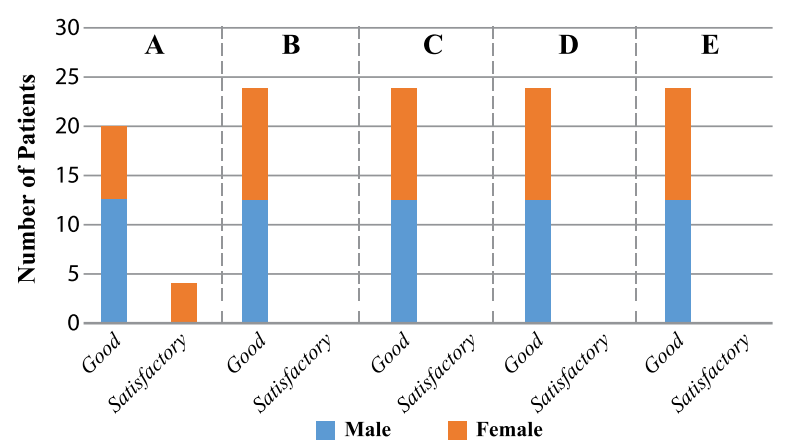

Fig 7 - Results of Major Ear Surgeries

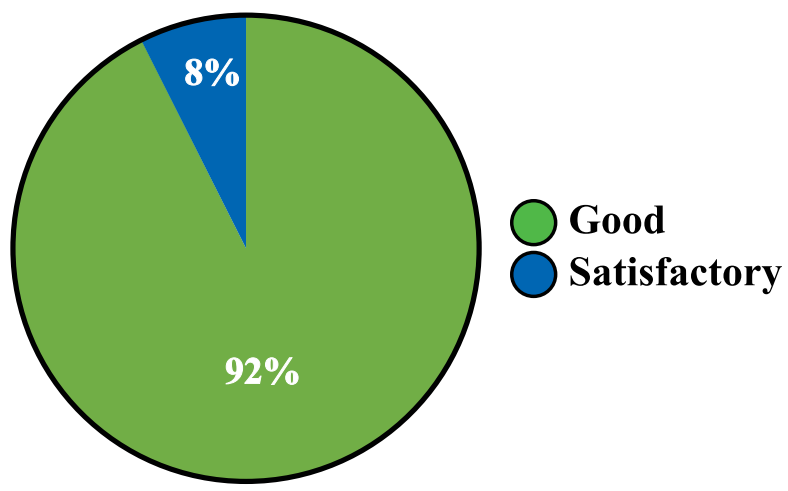

Fig 8 - Overrall Score of Ear Surgeries 
Tennekoon D., McPartlin D., Beynon Philips A., Tadikonda R., Arotiowa M. Patient Satisfaction on Post Operative Instructions Given by Hospital Staff on Discharge Volume : 6; Issue 1; pp 15-22

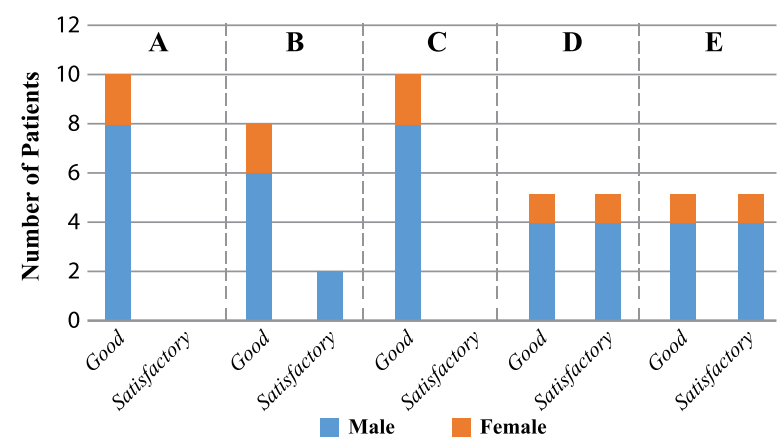

Fig 9 - Results of Minor Oropharynx Neck Surgeries

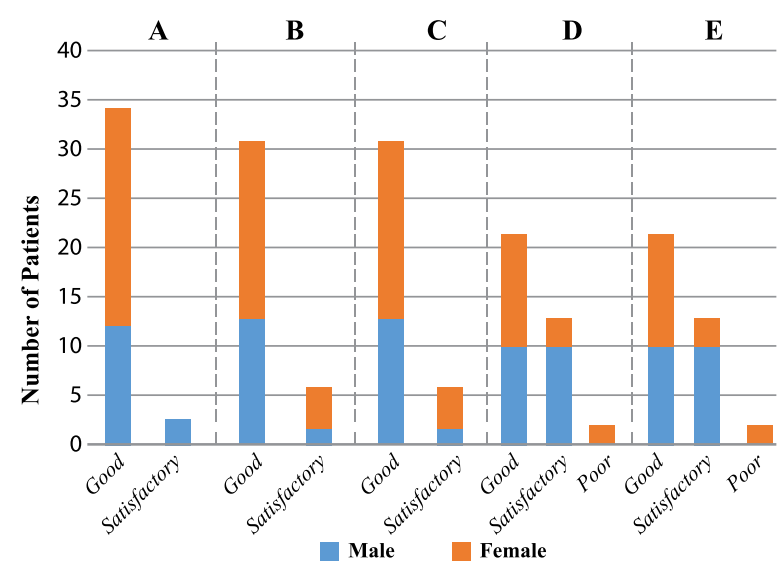

Fig 10 - Results of Intermediate Oropharynx and Neck Surgeries

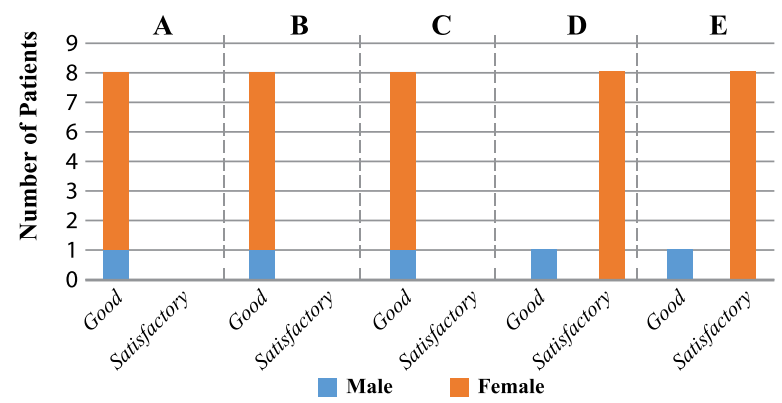

Fig 11 - Results of Major Oropharynx and Neck Surgeries

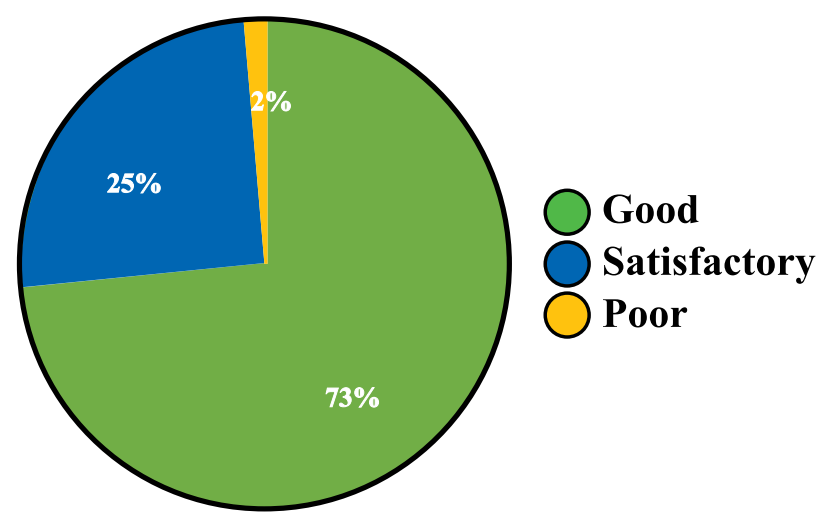

Fig 12- Overrall score for Oropharynx and Neck Surgeries

\section{Legend for Illustrations}

\section{Financial disclosures}

None of the authors have a financial interest in any of the products, devices, or drugs mentioned in this manuscript.

\section{Competing interests}

No competing interests. 